\title{
Proptosis, Orbital Pain, and Long-Standing Monocular Vision Loss Resolved by Surgical Resection of Intraosseous Spheno-Orbital Meningioma: A Case Report and Literature Review
}

\author{
Jonathan M. Parish ${ }^{10}$ Michael Shields ${ }^{2}$ Mackenzie Jones ${ }^{2}$ Scott D. Wait ${ }^{1,2}$ Vinay R. Deshmukh ${ }^{1,2}$ \\ ${ }^{1}$ Department of Neurological Surgery, Carolinas Medical Center, \\ Charlotte, North Carolina, United States \\ ${ }^{2}$ Carolina Neurosurgery and Spine Associates, Charlotte, North \\ Address for correspondence Vinay Deshmukh, MD, FACS, Carolina \\ Neurosurgery and Spine Associates, 225 Baldwin Ave, Charlotte, \\ NC 28204, United States (e-mail: vinay.deshmukh@cnsa.com).
} Carolina, United States

J Neurol Surg Rep 2020;81:e28-e32.

\begin{abstract}
Background and Importance We present a case of a patient with a residual intraosseous sphenoid wing meningioma presenting with proptosis, orbital pain, and monocular vision loss for 8 months who underwent decompression of the optic canal, orbital contents, and orbital reconstruction resulting in significant improvement in her vision loss with full resolution of proptosis and orbital pain.

Clinical Presentation A 43-year-old female presented with a 1 year history of headache, peri-orbital pain, proptosis, and severe vision loss. She had previously undergone subtotal resection of a large Simpson Grade 1 spheno-orbital meningioma 3 years prior at an outside institution. Workup at our institution revealed hyperostosis of the left greater wing of the sphenoid bone and narrowing of the optic canal along with bony enhancement concerning for residual tumor. The patient was given the recommendation from outside institutions for radiation, presumably due to the chronicity of her visual loss. Our institution recommended resection of the residual osseous tumor with orbital reconstruction. Less than 2 weeks after surgery, the patient noted significant improvement in orbital pain and vision. At 3 months, she had regained full and symmetric orbital appearance with no orbital pain. Her visual

Keywords

- craniotomy

- intraosseous meningioma

- vision loss acuity improved to 20/30 with full visual fields.

Conclusion Surgical decompression of the optic canal and orbital contents for tumor related sphenoid wing hyperostosis should be strongly considered, despite an extended duration of visual change and loss. This case report shows that vision can be significantly restored even after symptoms have been present for greater than 6 months.
\end{abstract}

\section{Introduction}

Primary extradural meningiomas are a rare form of meningioma occurring in less than $2 \%$ of cases. ${ }^{1}$ Of these primary extradural meningioma, intraosseous meningiomas constitute two thirds and when they are located in the sphenoid bone are known to commonly present with proptosis, orbital pain, and/or vision loss. Treatment options include surgical

received

July 14, 2019

accepted

September 28, 2019
DOI https://doi.org/

10.1055/s-0040-1708845. ISSN 2193-6358. gross total resection (GTR) or radiation therapy or a combination of the two. Numerous case reports show the benefit of GTR of spheno-orbital meningiomas in restoring visual function and ameliorating symptoms from mass effect on the orbital contents. ${ }^{2-4}$ Furthermore, improvement in long-standing visual dysfunction has been reported after surgical decompression of the optic nerve. ${ }^{3-9}$ In cases where severe visual dysfunction or blindness for greater than 6 months has

(c) 2020 Georg Thieme Verlag KG

License terms

Stuttgart · New York

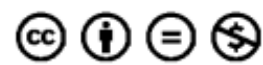




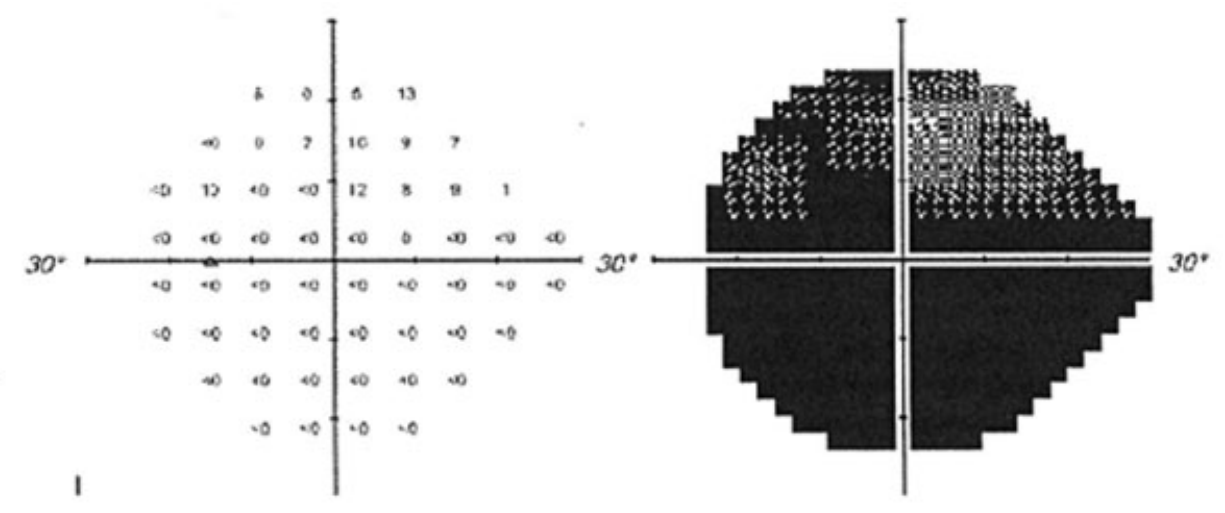

Fig. 1 Preoperative left visual field analysis.

occurred, it is generally considered irreversible and even optic nerve sacrifice has been advocated. ${ }^{10}$ Importantly, existing literature describes GTR of tumors that are predominantly soft-tissue based with only mild-to-moderate bony changes around the orbit. There are no case reports to our knowledge that address the management of long-term vision loss, proptosis, and orbital pain secondary to spheno-orbital meningioma present predominantly in the intraosseous space.

\section{Clinical Presentation}

\section{Onset and Course}

A 43-year-old female presented to an outside institution in July 2013 with headaches and was found to have a sphenoorbital meningioma with a large portion of the tumor extending into the extra-axial temporal convexity. She underwent craniotomy for meningioma resection and had improvement in her headaches. Comparison of pre- and postoperative films after her first resection showed good resection of the soft tissue component of the tumor but no resection of the bony portion. She did well until 3 years after initial surgery when she developed progressive headaches, proptosis, and visual loss. She presented to our institution in February of 2017 by which point she had constant eye pain and her vision had deteriorated to near blindness for the prior
7 to 8 months. A computed tomography (CT) and magnetic resonance imaging (MRI) of the brain showed progression of intraosseous spheno-orbital meningioma with significant hyperostosis and compression of the orbital content and optic canal. During formal ophthalmologic evaluation, she was not only able to count fingers directly in front of her face in her superior nasal visual field but also able to discern movement in the other visual fields. Her visual field index in her left eye was determined to be $5 \%$ (-Fig. 1). Her extraocular muscles were intact and she had an afferent pupillary defect in her left eye.

\section{Preoperative Radiological Findings}

CT of the head revealed abnormal thickening and sclerosis in the greater wing of the sphenoid bone and orbital apex, including evidence suggesting new bone formation (-Fig. 2). MRI of the brain showed abnormal thickening in the anterior aspect of the greater sphenoid wing with diminished T1 signal (-Fig. 3). Findings were suggestive of an intraosseous component measuring $2.3 \times 2 \mathrm{~cm}$. Expansion of the greater sphenoid wing contributed to orbital apex narrowing and compression of the left optic nerve. These findings suggested a primarily intraosseous meningioma recurrence. Due to the duration of her visual loss and predominantly intraosseous location of the tumor, multiple outside institutions recommended radiation
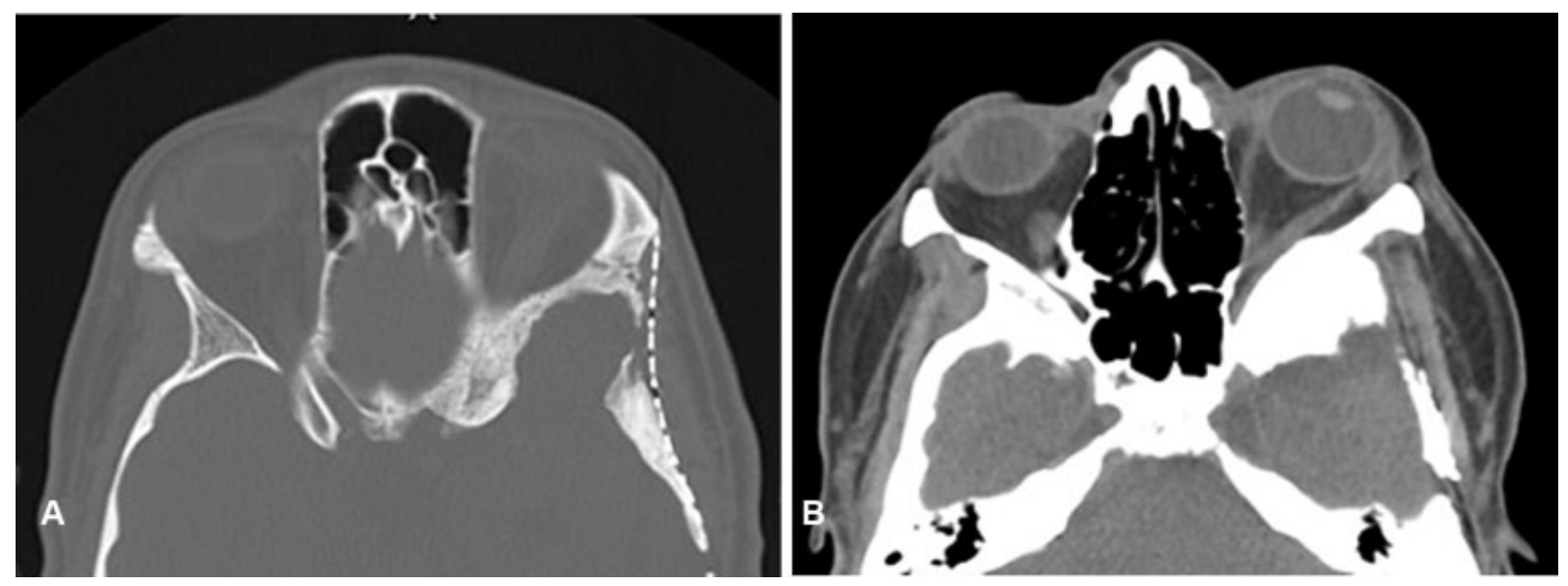

Fig. 2 (A) Preoperative computed tomography (CT) of left orbital invasion, significant hyperostosis, and proptosis: bone window image. (B) Preoperative CT of left orbital invasion, significant hyperostosis, and proptosis: soft tissue window image. 

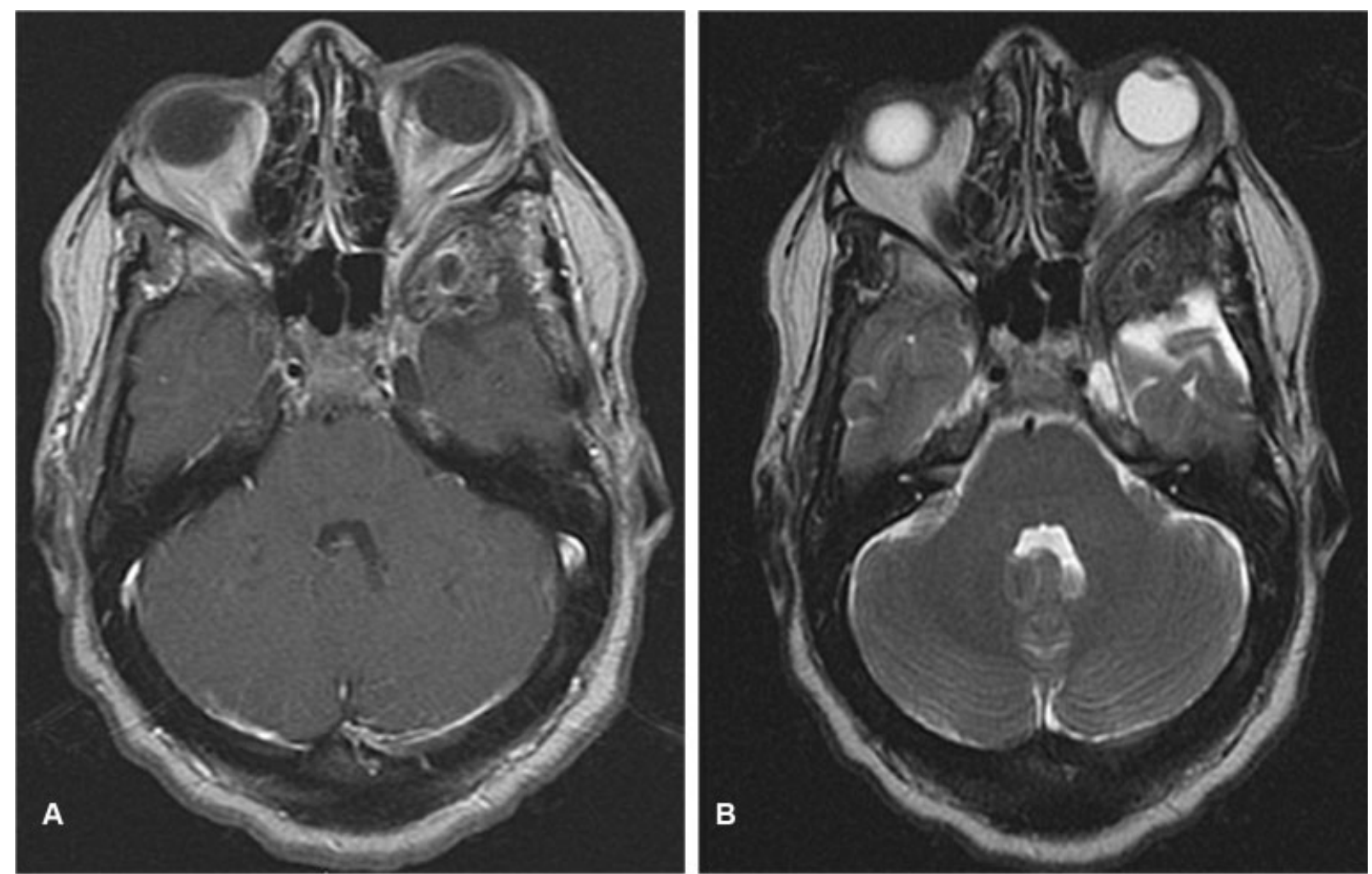

Fig. 3 (A) Magnetic resonance imaging (MRI) T1-weighted post-contrast image revealing enhancing tumor and optic nerve compression. (B) MRI T2-weighted images revealing proptosis and optic nerve compression

therapy. Surgery was recommended by our institution. At the time of redo-surgery, the patient had 8 months of near complete visual loss

\section{Operative Technique}

The goal of surgery was GTR of the intraosseous tumor with full decompression of the optical canal to alleviate orbital pain and proptosis of the left eye and restore visual function. An orbitozygomatic and left pterional craniotomy was performed for exposure of the anterior cranial fossa, middle cranial fossa, and paraclinoidal region. Involved portions of the lesser and greater wings of the sphenoid (including the lateral orbital wall) were resected. Resection of the greater wing of the sphenoid at the floor of the temporal fossa was taken back to the medially located foramina. The optic nerve was completely decompressed within the optic canal from the falciform ligament to the annulus of Zinn, which was greatly facilitated by intradural anterior clinoidectomy with optic strut resection. The superior and lateral orbital walls were then reconstructed with mesh ( - Fig. 4).
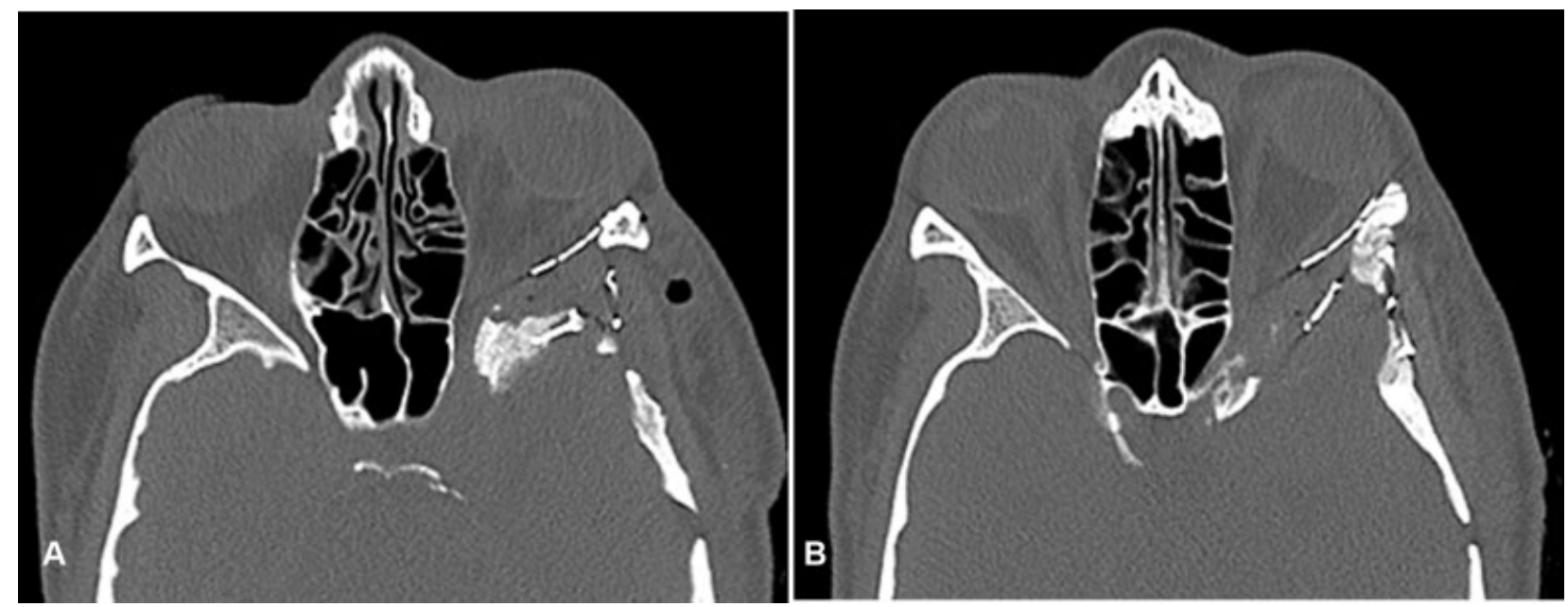

Fig. 4 (A) Postoperative computed tomography (CT) shows reconstruction of orbit and improvement in proptosis. (B) Postoperative CT shows reconstruction of orbit and improvement in proptosis. 


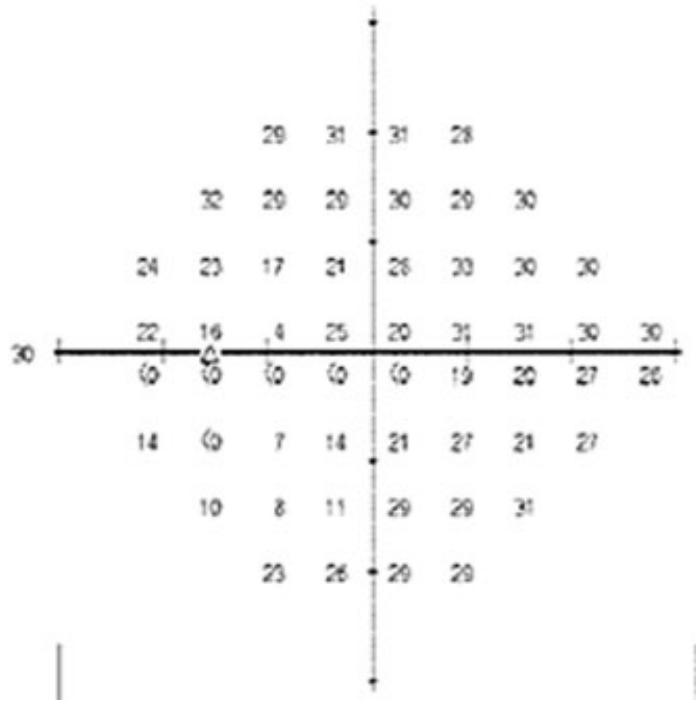

Fig. 5 Postoperative left visual field analysis.

\section{Postoperative Course}

There were no major postoperative complications. Within 2 weeks of surgery, the patient noted significant improvement in her preoperative symptoms with functional vision in her left eye, improvement in pain, and resolution of proptosis. At 2 months, her visual acuity in her left eye was 20/40 with full visual fields and eye movements consistent with mild optic neuropathy (-Fig. 5). She also reported full resolution of orbital pain and decrease in frequency of headaches.

\section{Discussion}

Mass effect secondary to tumors of the orbit, tuberculum sellae, and sphenoid wing can compress the orbital contents and optic canal, resulting in proptosis, eye pain, headaches, and visual loss. ${ }^{11-15}$ In particular, sphenoid wing meningiomas with hyperostosis present most commonly with proptosis followed by visual loss and headaches, with rates of 71 , 59 , and $42 \%$, respectively in one series. ${ }^{4}$

Visual acuity is affected in 40 to $60 \%$ of spheno-orbital meningiomas ${ }^{16,17}$ and length of compression has been closely associated with visual acuity outcome. Schick and Hassler ${ }^{3}$ reported on 53 patients with tuberculum sellae meningiomas treated over an 11-year period. Preoperative duration of symptoms was negatively correlated with preoperative visual acuity and postoperative recovery of visual deficits was worse in patients with symptoms longer than 6 months. Similar findings were noted by Zevgaridis et $\mathrm{al}^{9}$ in 62 patients with sellar meningiomas. Visual prognosis was favorably affected by duration less than 7 months $(p<0.037)$. Though different in anatomic location of origin, the mechanism of optic nerve compression is analogous to our case and serves as a reasonable comparison.

There have been prior reports in the literature demonstrating that severe visual deficit and even blindness can be improved after optic nerve decompression. Oya et $\mathrm{al}^{8}$

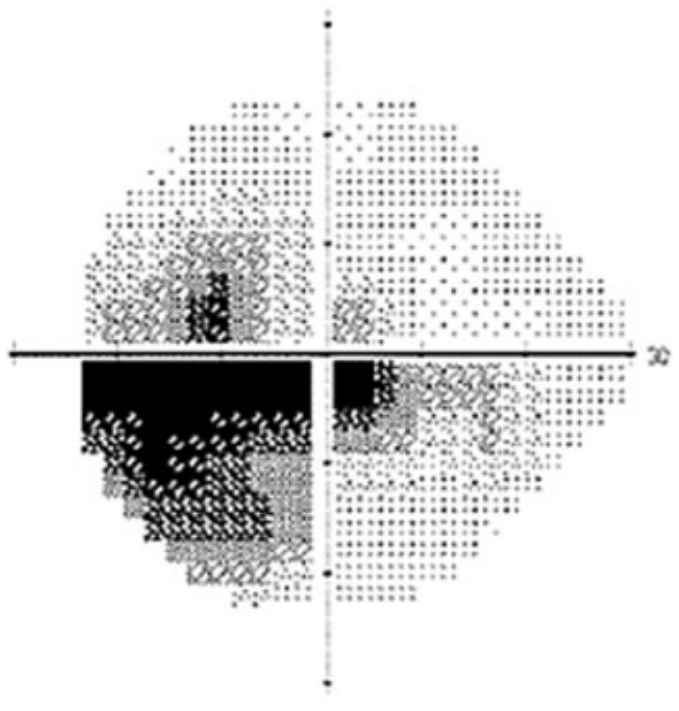

reported on 39 patients with spheno-orbital meningiomas of which four patients had severe vision loss (defined as $20 / 200$ or worse) and two had blindness. Neither patient with blindness had functional recovery of vision, though two patients with severe vision loss of 12 months had functional recovery of vision to mild (defined as 20/30-$20 / 100$ ) or no symptoms. Tamura et $\mathrm{al}^{4}$ reported an innerthird sphenoid wing meningioma in a 65-year-old female with progressive vision loss for over a year with complete blindness of 6 months that improved after decompression. Preoperatively the patient had an intact pupillary light reflex but no light discrepancy. The patient did recover visual acuity with the exception of a central scotoma. These studies also support surgical decompression regardless of extent of visual deficit.

In our case, the duration of visual symptoms was 1 year with 8 months of severe vision loss and near vision limited to finger discrimination only. The primarily intraosseous nature of this tumor with the significant hyperostosis of the bony orbital structures led us to believe that surgical resection with decompression of optic canal and orbital contents represented the best option for improvement in the patient's presenting symptoms. We did not feel that radiation alone would have resulted in the rapid recovery of vision and near complete resolution of proptosis and orbital pain.

\section{Conclusion}

Our case represents the most significant postoperative improvement in severe vision loss related to intraosseous meningioma found in the literature, making a strong for surgical decompression of the bony optical canal and orbital contents even in cases where vision loss is chronic. This case report shows that vision can be significantly restored even after visual symptoms have been present for greater than 6 months and even in cases of primarily bony compression of the optic nerve. 


\section{e32 Intraosseous Meningioma with Vision Loss Parish et al.}

\section{Conflict of Interest}

None.

\section{Acknowledgment \\ Acknowledgment of contribution: Ishaan Nandwani.}

\section{References}

1 Lang FF, Macdonald OK, Fuller GN, DeMonte F. Primary extradural meningiomas: a report on nine cases and review of the literature from the era of computerized tomography scanning. J Neurosurg 2000;93(06):940-950

2 Hussaini SM, Dziurzynski K, Fratkin JD, Jordan JR, Hussain SA, Khan M. Intraosseous meningioma of the sphenoid bone. Radiol Case Rep 2015;5(01):357

3 Schick U, Hassler W. Surgical management of tuberculum sellae meningiomas: involvement of the optic canal and visual outcome. J Neurol Neurosurg Psychiatry 2005;76(07):977-983

4 Tamura R, Takahashi S, Horikoshi T, Yoshida K. Improvement of long-term blindness caused by compression from inner-third sphenoid wing meningioma after optic canal decompression: an extremely rare case report. Surg Neurol Int 2016;7:67

5 Chicani CF, Miller NR. Visual outcome in surgically treated suprasellar meningiomas. J Neuroophthalmol 2003;23(01):3-10

6 Galal A, Faisal A, Al-Werdany M, El Shehaby A, Lotfy T, Moharram $H$. Determinants of postoperative visual recovery in suprasellar meningiomas. Acta Neurochir (Wien) 2010;152(01):69-77

7 Mariniello G, Bonavolontà G, Tranfa F, Maiuri F. Management of the optic canal invasion and visual outcome in spheno-orbital meningiomas. Clin Neurol Neurosurg 2013;115(09):1615-1620

8 Oya S, Sade B, Lee JH. Sphenoorbital meningioma: surgical technique and outcome. J Neurosurg 2011;114(05):1241-1249
9 Zevgaridis D, Medele RJ, Müller A, Hischa AC, Steiger HJ. Meningiomas of the sellar region presenting with visual impairment: impact of various prognostic factors on surgical outcome in 62 patients. Acta Neurochir (Wien) 2001;143(05):471-476

10 Park SC, Lee SH. Penetration and splitting of optic nerve by tuberculum sellae meningioma. J Korean Neurosurg Soc 2016; 59(05):525-528

11 Margalit NS, Lesser JB, Moche J, Sen C. Meningiomas involving the optic nerve: technical aspects and outcomes for a series of 50 patients. Neurosurgery 2003;53(03):523-532, discussion 532-533

12 Newman SA, Jane JA. Meningiomas of the optic nerve, orbit and anterior visual pathways. In: Al Mefty O, ed. Meningiomas. New York: Rave Press; 1999:461-494

13 Nozaki K, Kikuta K, Takagi Y, Mineharu Y, Takahashi JA, Hashimoto N. Effect of early optic canal unroofing on the outcome of visual functions in surgery for meningiomas of the tuberculum sellae and planum sphenoidale. Neurosurgery 2008;62(04): 839-844, discussion 844-846

14 Taha AN, Erkmen K, Dunn IF, Pravdenkova S, Al-Mefty O. Meningiomas involving the optic canal: pattern of involvement and implications for surgical technique. Neurosurg Focus 2011;30 (05):E12

15 Agrawal V, Ludwig N, Agrawal A, Bulsara KR. Intraosseous intracranial meningioma. AJNR Am J Neuroradiol 2007;28(02): 314-315

16 Mariniello G, Maiuri F, Strianese D, et al. Spheno-orbital meningiomas: surgical approaches and outcome according to the intraorbital tumor extent. Zentralbl Neurochir 2008;69(04): 175-181

17 Ringel F, Cedzich C, Schramm J. Microsurgical technique and results of a series of 63 spheno-orbital meningiomas. Neurosurgery 2007;60(4, Suppl 2):214-221, discussion 221-222 